\title{
Desarrollos teóricos en contabilidad
}

\author{
Hárold Álvarez Álvarez \\ Profesor e investigador Universidad Autónoma Latinoamericana \\ y Universidad de Manizales - Colombia
}

\section{Resumen}

Este artículo realiza varias reflexiones alrededor de la evolución teórica de la contabilidad, para ello hace alusión a perspectivas metodológicas empleadas por otros autores, tales como paradigmas y programas de investigación. Se habla de las principales doctrinas clásicas de la contabilidad y de algunas contemporáneas, todas ellas desde la perspectiva funcionalista. El artículo se encuentra orientado hacia la formación básica en teoría contable, de forma que puede ser apropiado para su uso como texto en el aula de clase. Además, se exalta el rol de la contabilidad como disciplina social y en alguna medida se describe su impacto en las organizaciones.

\section{Palabras clave}

Doctrinas contables, escuelas contables, programas de investigación, paradigmas, tendencias positivas y normativas, historia contable.

\section{Abstract}

This paper makes different reflections around the theoretical evolution of accounting, for that, it makes allusion to methodological perspectives used by other authors, such as paradigms and investigation programs. It talks about the main classical doctrines of accounting and some contemporary, all of them from a functionalist perspective. The article is oriented to the basic formation in the accounting theory, which can be appropriate to use it as a text in the class room. It also exalts the role of accounting as a social discipline and in some way its impact in the organizations is described.

\section{Key words}

Accounting doctrines, investigation programs, paradigms, positive and normative tendencies, accounting history.

Recibido: N.A. - Versión final aceptada: N.A. - Autor invitado 


\section{Introducción}

El objetivo de este artículo es la divulgación descriptiva de algunas propuestas de análisis y clasificación de las experiencias vividas por los contables en el proceso de construcción disciplinar de la contabilidad. Como base de la descripción, se han seleccionado diversos trabajos de connotados autores, fundamentalmente europeos, que han realizado sus reconstrucciones históricas a la luz de enfoques socio-epistemológicos, en particular de los planteamientos de Popper, Kuhn y Lákatos, que para auscultar la experiencia investigativa teórica de cualquier disciplina constituye una herramienta invaluable.

Dos vías han estado en discusión para abordar la temática que hoy nos ocupa, con el fin de establecer el desarrollo histórico reciente de la reflexión contable. Aquél que predica el desarrollo del conocimiento por evolución progresiva o el contrapuesto que predica el carácter discontinuo de tal proceso. Hemos escogido textos de autores que se orientan por la segunda alternativa a través de la cual se considera a la historia no como simple crónica de los acaecimientos que se han ido sucediendo en el tiempo, sino como análisis científico del hecho histórico.

Cuando en su introducción a La estructura de las revoluciones científicas (1969), Kuhn se refiere al papel de la historia y del historiador en el estudio de la ciencia dice:

Tradicionalmente al historiador se le ha encargado determinar por qué hombre y en qué momento fue inventado o descubierto un hecho, ley o teoría; y describir y explicar el conjunto de errores, mitos y supersticiones que impidieron una acumulación más rápida de los componentes del caudal científico moderno. Sin embargo, durante los últimos años, unos cuantos historiadores de la ciencia han descubierto que les es cada vez más difícil desempeñar las funciones que el concepto del desarrollo por acumulación les asigna.

La ciencia vista como proceso histórico discontinuo nos permite observar teorías y creencias que en el momento se han considerado como científicas pero que con el tiempo resultan incompatibles con las que posteriormente adquieren consistencia y son objeto de considera- 
ción. No es que aquellas pierdan el carácter de científicas, pero el hecho de haber sido descartadas hace difícil poder considerar el proceso de desarrollo científico con carácter acumulativo.

Además del carácter discontinuo del proceso científico es necesario anotar que dicha tarea no corresponde a la actividad aislada, solitaria o esotérica de un iluminado. La ciencia es una actividad comunitaria sometida a la influencia del entorno en el que se desarrolla y al que pretende servir una vez ha coronado su objetivo, el trabajo científico.

Esta nueva forma de ver el quehacer de la ciencia, con elementos sociológicos de por medio, en donde no es extraño identificar intereses, valores, pasiones etc., como en cualquier empresa humana, se origina en los trabajos de Popper, Kuhn, Lákatos, Bunge, Hanson y otros. Este enfoque nos permite analizar la tarea de la ciencia con una óptica más realista y desde diversos puntos de vista, que en todo caso constituyen una ganancia para comprender el surgimiento, la prevalencia, la coexistencia, la rivalidad y la declinación de teorías, tanto como el resultado del trabajo de las comunidades, que se agrupan a su alrededor y conviven con ellas.

La construcción lakatosiana de Programas de investigación, como una conjunción enriquecida de los planteamientos popperianos y kuhnianos nos servirá como hilo conductor de esta reflexión sobre el proceso de construcción disciplinar contable, que de antemano, advertimos, está plegado de intereses regulativos, normativos, que en muchas ocasiones han producido formulaciones enmarcadas dentro el concepto denominado "teorías del falso conocimiento" o conocimiento interesado. Éste está conformado por postulaciones tautológicas que se auto validan sin necesidad de referencia empírica, con lo que las construcciones teóricas derivadas no son otra cosa que convencionalismos y/o especulaciones sobre la realidad. Dentro de este debate han surgido posiciones, desde las positivistas a ultranza hasta las defensoras extremas de la normatividad absoluta, circunstancias que han originado tendencias investigativas disímiles que han influenciado con sus teorías los entramados regulativos contables de países y grupos de naciones en los últimos treinta años.

En primer término nos referiremos a varios trabajos de los profesores Vicente Montesinos, Leandro Cañibano, Jorge Tua, Richard Mattessich, Leandro Cañibano, y José Antonio Gonzalo, en la óptica de 
identificar el quehacer reflexivo contable a la luz de la metodología de "programas y subprogramas de investigación", o a partir de enfoques similares.

También nos referiremos a diversos avances teóricos entre los que tocaremos el tema relativo a la formalización del pensamiento contable como uno de los elementos básicos de la disciplina en el proceso de construcción de su discurso científico, haciendo mención de la insoslayable producción del profesor Richard Mattessich, así como de algunos de los aportes de los profesores Watts y Zimmerman, Coase, Simon, Jensen y Meckling, Cañibano y Moisés García. Estos aportes se detallarán aprovechando las referencias que a los mismos se hacen en el trabajo de los profesores Cañibano y Gonzalo (1995).

Finalmente, en la conclusión, expresaremos nuestros propios puntos de vista críticos del proceso de desarrollo disciplinar y propondremos algunos planteamientos que consideramos necesarios para el avance de desarrollo disciplinar.

\section{Programas y subprogramas de investigación}

Las décadas de los sesenta y los setenta se manifiestan como los tiempos que propiciaron cambios cualitativos de la forma de ver el conocimiento contable y, por múltiples circunstancias, algunas de las cuales mencionaremos más adelante, los teóricos de la contabilidad se acercaron a disciplinas humanísticas y filosóficas para comprender mejor los procesos de avances, retrocesos y estancamiento de la producción teórica básica y de sus derivaciones, tanto en el nivel nacional como internacional.

De este cambio gestáltico en el análisis de la historia contable surgieron los trabajos de los profesores Cañibano, Tua, Montesinos y de otros no menos importantes, influenciados por la propuesta lakatosiana de programas de investigación que, en propias palabras del profesor Montesinos (1978), significó:

La metodología de los programas de investigación se sitúa en el ámbito del desarrollo del conocimiento científico, y supone un planteamiento diacrónico o dialéctico frente a las metodologías 
sincrónicas, estructurales o intemporales, propias del que se ha venido a denominar el contexto de justificación. La metodología de los programas de investigación se preocupan de las génesis de las teorías y por la lógica de su evolución y desarrollo, guiada por una "heurística positiva", en cuyo establecimiento juegan un importante papel los estudios de historiografía, tanto interna como externa a la ciencia de la Contabilidad... La metodología de Lákatos pone en marcha igualmente una heurística negativa, basada en el "modus tollens", que sin embargo no se aplica a las hipótesis constitutivas del "núcleo firme" del Programa de investigación, aunque sí a las hipótesis auxiliares en el denominado "cinturón protector"... La metodología comentada permite agrupar las teorías en torno a diversos paradigmas aceptados convencionalmente (influencia kuhniana) formados por un conjunto de hipótesis fundamentales. De este modo conseguimos evitar los desenfoques conceptuales que pudieran derivarse de una consideración desordenada y arbitraria de las diversas teorías.

Una teoría lleva la impronta del Programa al que pertenece y su cuestionamiento está condicionado por la solidez de dicho Programa, el cual es abandonado cuando su productividad desaparece en el proceso de confrontación problemática y si ha surgido un nuevo Programa alternativo que dé cuenta de los problemas solucionados y no solucionados por aquél.

Cuando un programa no predice anomalías o no se resuelve los nuevos problemas identificados se dice que ha entrado en crisis, es decir, en un estadio degenerativo. "Esta degeneración en el caso de las teorías positivas, puede interpretarse como pérdida de potencialidad para predecir hechos nuevos y, en el caso de las teorías normativas, como incapacidad para prescribir normas que resulten en una superior eficiencia de las acciones".

Bajo esta óptica los profesores antes mencionados han identificado a lo largo de la historia de la contabilidad y, especialmente, en los lapsos catalogados como "periodos científicos", diversas construcciones que ha identificado como programas o subprogramas de investigación sucesivos o alternativos, ya que en algunos casos han sucedido y en otros han coexistido en diversos contextos espacio-temporales. 


\section{La perspectiva de Montesinos}

Para el profesor Montesinos (1978), cabe resaltar los periodos siguientes:

- Empírico, que comprende desde la Antigüedad hasta la alta Edad Media, aproximadamente el inicio del siglo XIII. Este largo periodo se caracteriza por la inexistencia de sistemas contables complejos, aunque sí se muestra a lo largo del mismo una permanente preocupación social por el mantenimiento de sistemas contables en coordinación con el desarrollo de las actividades económicas. Se encuentran ejemplos de anotaciones que datan desde la más remota antigüedad, especialmente de los sumerios, egipcios, griegos y romanos. A raíz de la invasión de los bárbaros se origina un periodo de regresión en la actividad económica en el Occidente cristiano, lapso del que prácticamente no queda vestigio de ninguna cuenta. Entretanto, en el Imperio Romano de Oriente, en el Egipto ptolemaico, romano y bizantino, se observa una progresiva evolución hacia la estructura medieval, conservándose cuentas hasta épocas próximas a la invasión islámica. A partir del siglo XI, con las Cruzadas, surge la actividad económica en Europa Occidental y con ello reaparecen los registros contables de tales actividades. Ésta es la etapa final del periodo empírico, dentro de la Alta Edad Media, en que se vive una época de progreso mercantil paulatinamente floreciente y en que se pasará, en un término relativamente reducido de tiempo, de los registros más primitivos, como los memoriales, pasando por la Partida Simple, hasta arribar a los inicios de la Partida Doble.

- Génesis y aparición de la Partida Doble, periodo comprendido entre el siglo XIII y finales del siglo XV. Este periodo comienza con la aparición del "Liber Abaci" de Leonardo Fibonacci de Pisa, que contempla temas de teneduría de libros y de matemáticas financieras desarrollados en números arábigos. Es en esta época cuando se asiste a la transformación de la Partida Simple en la Partida Doble, gracias al auge comercial y a la aparición del capitalismo, cuyo espíritu está movido por una nueva idea domi- 
nante: el tránsito del concepto de riqueza al de capital productivo, es decir, un capital que en el proceso de mantenerse origina un capital adicional, produciendo así mismo una racionalidad en las actividades ordinarias de la sociedad que, a su vez, el nuevo sistema de registro contribuye a consolidar. La expansión del comercio, el desarrollo de las operaciones a crédito, el nacimiento de las sociedades comerciales y la representación comercial bajo la forma de contratos de comisión o de mandato, figuran entre los factores que contribuyeron al desarrollo de la contabilidad en esta época. Aparecen en este periodo otras preocupaciones que para su desarrollo definitivo e integrado habrá que esperar al siglo XX, pero cuyos primeros planteamientos surgen en esta época; se trata de la contabilidad de costos, que de sus elementales desarrollos existen vestigios en la contabilidad de Francesco Dantini, de Prato, Italia, de 1390; así mismo, en los registros de la casa de los Médicis, del siglo XV, se utilizan técnicas de cálculo de costos.

- Expansión y perfeccionamiento de la Partida Doble. Doctrina del Contismo, cuyos desarrollos comprenden los siglos XVI hasta mediados del siglo XIX. Aunque es de aceptación común que durante este periodo los avances metodológicos no fueron de gran importancia, si son significativos los avances en la aplicación de la nueva orientación a través de las fronteras geográficas, así como los desarrollos de los principios de periodización y de valoración de inventarios. Es de destacar la presencia, en Italia, al inicio de este periodo, del reconocido autor Ángelo Pietra, quien complementa la obra de Paciolo al incluir nuevos conceptos contables tales como la contabilidad presupuestal, la distinción entre empresas y propietario, y el perfeccionamiento del balance de comprobación. Un siglo después aparece la obra del autor Ludovico Flori que aporta, en desarrollo de los planteamientos de Pietra, la idea de la existencia de tres estados contables, el de Resultados, el de Balance y el de Capital. Merece especial consideración la obra de Menher, en los Países Bajos, en el siglo XVI, en la que se pronuncia por una contabilización de los inventarios al valor de realización o corriente, y al valor de costo o de adquisición para los bienes de capital. 
- El profesor Montesinos anota con respecto al desarrollo de la contabilidad en la Francia de esta época: "la escuela francesa de los siglos XVII y XVIII va elaborando progresivamente una teoría de la contabilidad entendida como ciencia de las cuentas, apartándose progresivamente de las preocupaciones patrimoniales que siempre habían caracterizado a las actividades contables, desde muchos siglos antes de la conformación del método de Partida Doble". Una de las fallas que se anotan a estos planteamientos es el confundir uno de los instrumentos contables, la cuenta, con la disciplina contable en general, con lo que se la reduce a, tan sólo, la descripción del funcionamiento de un sistema de cuentas. Debe destacarse la importante posición que ganó la cuenta de inventarios en la teneduría de libros, y su papel en la determinación de la renta, como consecuencia del desarrollo del método de Partida Doble. Así mismo, en esta época, se establecen los períodos contables fijos, en un principio de dos años, aunque no es clara la periodifización de los factores de producción ni tampoco la contabilización de la depreciación. Se inicia la aplicación del criterio de prudencia, materializado en la contabilización del valor más bajo, ello influenciado por las quiebras fraudulentas ocurridas en esos tiempos en Francia. Debe mencionarse la obra de Matice de la Porte, de 1772, La ciencia de los negocios y la teneduría de libros, en la que se plantean cuestiones prácticas de contabilidad en aplicación de los planteamientos de Paciolo, Pietri y Flora y que, además, aportan una distinción importante entre la personalidad moral de la empresa y la del propio propietario, fijando así la base de la denominada Teoría de la empresa. Finalmente merece especial mención el autor Edmund Desgranes de Randy quien con su propuesta de dividir las cuentas generales en cinco (caja, mercancía, efectos a recibir, efectos a pagar y pérdidas y ganancias), suscita la famosa escuela Cinco cuentista; aunque la obra de Desgranes tuvo gran influencia, no pasa de ser una buena vulgarización de los conceptos contables de los anteriores tratadistas ya mencionados.

- Científico, comprendido entre 1840 y nuestros días. En tal sentido seguimos, en apretado resumen, al profesor Vicente Montesinos, quien advierte posibles deformaciones de la realidad 
cuando se trata de clasificar en ciertas corrientes doctrinales la obra de algún autor influenciado por más de una corriente de pensamiento.

Las corrientes doctrinales contables que, según el profesor Montesinos, abarcan el desarrollo investigativo, desde mediados del Siglo XIX y se extienden hasta el siglo XX, son las siguientes:

a) Doctrinas jurídico-personalistas.

b) Doctrinas contestas y neocontistas.

c) La teoría matemática y el empeño formalizador.

d) La introducción de los aspectos conductistas y la percepción comunicacional.

e) El enfoque económico.

Naturalmente estas alternativas no son excluyentes, de manera que pueden coexistir en el tiempo y en el espacio, todo ello dependiendo de las características de desarrollo socioeconómico de los ámbitos de las organizaciones relativas a las cuentas.

a) Doctrinas jurídico-personalistas. Esta corriente está basada en la Teoría de la personificación, a través de la cual se pretende explicar el funcionamiento de la cuentas considerando que todas ellas representan obligaciones del propietario. Esta doctrina, cuyos antecedentes datan de dos siglos atrás, fue perfeccionada por la Escuela Toscana, que con la obra de Giuseppe Cerboni, avanza hacia una doctrina de las responsabilidades personales entre los administradores de la organización económica.

Para Cerboni la contabilidad es la ciencia de las funciones, de las responsabilidades y de las cuentas administrativas de las haciendas, distinguiendo dentro de su contenido cuatro diferentes, así:

1. Estudio de las funciones de la administración económica de las empresas con el fin de determinar las leyes naturales o civiles según las cuales las mimas se explanan y se regulan.

2. Organización y disciplina interna de las empresas.

3. Cálculos, o sea la aplicación de la matemática a los hechos administrativos y su demostración. 
4. Estudio de los métodos de registración destinados a coordinar y representar hechos administrativos de las empresas, en punto a revelar los procesos y sus hechos específicos, jurídicos y económicos, y mantenerlos reunidos todos en una sola ecuación.

Cerboni pretendió dotar a la contabilidad de una forma científica, por lo que propuso una estructura de verdad para la disciplina basada en seis axiomas, cada enunciado con uno o más corolarios con los que pretendió establecer unas especies de leyes de comportamiento entre los actores empresariales y la información contenida en la contabilidad. Esta construcción no resiste la crítica ante un examen epistemológico riguroso, pero tiene el mérito de ser el primer precedente que intenta cumplir con requisitos formales en la edificación de teorías científicas en el campo de la contabilidad.

Cerboni publicó una memoria en 1873 titulada Primi saggi di logismografia, que tuvo extraordinaria acogida. La logismografia, cuyo significado es el de una descripción razonada de las cuentas es la más moderna expresión de la teoría personalista, visión jurídico-administrativa que considera a la entidad económica, "empresa", desde el ángulo de las relaciones jurídicas resultantes de la actividad de las personas que están encargadas de su gestión y administración y de las terceras personas interesadas en ella.

Hubo muchos seguidores de esta teoría, entre los que se destacan Rossi, Bonalumi, Riva Gitti, Mondini en Italia; Faure en Francia; Fulton, Cronhelm, Sprague y Kester en Estados Unidos fueron seguidores de la Ownership theory, de raigambre jurídico-personalista.

Dado que cualquier actividad económica está inserta en un marco jurídico de referencia la estructura de tal ámbito condiciona los procesos objeto de la contabilidad, así como los profesionales contables que le ejecutan. En tal sentido muchos de los planteamientos cerbonianos no responden a las necesidades disciplinares y que son ficciones que no reflejan la realidad factual en la que está inmersa la contabilidad. Por ello surgen, al margen de dichos planteamientos, trabajos que se agrupan en diversas líneas, como la mencionada Teoría del propietario, que luego deriva en la reconocida tendencia denominada Teoría de la entidad, que tiene su origen en la separación de la empresa y el propietario. 
b) Doctrina contista. Considerada como la primera escuela aparecida en la disciplina contable, está basada en la Teoría de la personificación, centrada en el funcionamiento de las cuentas y cuyo mecanismo trataba de explicarse a través de la ficción de la personificación.

Esta escuela admitía que las cuentas son el objeto de la contabilidad. Sus teorizaciones primeras fueron desarrolladas por Nicoló D’Anastasio en 1803 y Giuseppe Bornachini, a partir de 1818, si bien sus primeros planteamientos prácticos datan del siglo XVII. Desde un punto de vista filosófico, los contistas no buscaron la "esencia", o sea, una indagación sincera sobre lo que es la cuenta. La cuenta como registro no llena condiciones de ser objeto científico; cosa diferente es "lo que la cuenta registra", que son fenómenos patrimoniales.

Para la escuela contista, la contabilidad es la ciencia de las cuentas y tiene por objeto establecer los procedimientos apropiados para realizar los adecuados registros en las mismas. Tales procedimientos son el resultado de una centenaria práctica desarrollada primero en Italia, y más tarde en la mayoría de los lugares que ostentaron auge comercial e industrial.

La doctrina neocontista se origina en los planteamientos de Fabio Besta, aunque su desarrollo y difusión se debe a autores no italianos, específicamente franceses y norteamericanos. Esta corriente rechaza la ficción de la personificación adoptada por el contismo clásico y, en su lugar, se preocupa por la noción de valor, captada y manifestada a través de las cuentas y de los balances. El neocontismo ve en la contabilidad algo más que un simple mecanismo puramente formal de registro y considera que es preciso conocer la realidad para poder gobernar económicamente una entidad a través de las cuentas y ellas, las cuentas, son su centro de interés.

El neocontismo tuvo notoria influencia en países del centro de Europa como Alemania, Suiza, Hungría, entre otros. También en Estados Unidos y Francia se manifestaron seguidores de esta corriente.

c) La teoría matemática y el empeño formalizador. El profesor Montesinos hace, de partida, la siguiente diferenciación: por teoría matemática se entenderá la corriente de pensamiento que considera a la contabilidad dentro de la categoría de ciencias formales, como una 
parte de las matemáticas; de otro lado, significa empeño formalizador la tendencia, cada vez más extendida, dirigida a exponer las teorías contables en términos formales, utilizando para ello el instrumental matemático.

Aunque desde el inicio de las formulaciones que se pueden catalogar de teorías, como la de Paciolo, varios matemáticos notables fueron tratadistas contables y utilizaron algunas fórmulas matemáticas en sus tratados contables, la teoría matemática propiamente dicha dentro de la contabilidad apareció sólo a finales del siglo XIX.

La teoría matemática considera que la contabilidad es un método de observación, construido de acuerdo con los principios del razonamiento matemático, e independiente de las características particulares del objeto o campo de observación al que se aplique.

Para autores como Léutey y Guilbault, la contabilidad es una rama de las matemáticas y su objeto sería el de redactar las cuentas con vista a comparar valores materiales. Como toda cuenta se apoya en operaciones aritméticas, se desprende de ello que la contabilidad es una rama de las matemáticas y que ha de clasificarse entre las ciencias exactas. Estos autores en la misma obra incluyen la siguiente definición de contabilidad que no es concordante con la anterior, pues se adscribe más a la ciencia económica: "la contabilidad, rama de las matemáticas, es la ciencia de la coordinación racional de las cuentas relativas a los productos del trabajo y a las transformaciones del capital; es decir, a las cuentas de la producción, de la distribución y del consumo de las riquezas privadas y públicas" (Montesinos, 1978).

En 1924 E. de Fages de Latour, publicó Los conceptos fundamentales de la contabilidad, en el que desarrolla una propuesta de contabilidad pura, percibida como un método abstracto de observación de fenómenos alejado de los contenidos empíricos de dichos fenómenos. "La contabilidad es la numeración de las unidades en movimiento", diría de Fages y ejemplifica con casos supuestos de contabilidad en ciencias naturales, como la biología animal. Esta perspectiva es reafirmada por P. Garnier cuando afirma: "El método tiene sus reglas, que nada deben al sujeto observado. No hay, no puede haber teoría económica de la contabilidad, como no hay teoría jurídica... Todavía aquí, más que nunca, recordamos que el método contable es solamente un método de observación, nada más" (Montesinos, 1978). 
El autor brasileño Francisco D’Auria (1949) formula en su obra la definición de contabilidad pura y sus aplicaciones: "La contabilidad, precisamente, define un objeto sometido a observación y elaboración, para su conocimiento pormenorizado de su estado inicial, de las variaciones en el tiempo, y del estado actual en cualquier momento... Todo puede ser objeto de aplicaciones de la contabilidad. Cualquier organismo material o moral, o cualquiera de sus partes es susceptible de contabilización" (Montesinos, 1978). Para D’Auria la contabilidad se aplica al conocimiento de los estados y de la evolución de los sistemas, entendidos como conjuntos armónicos, de los más simples a los más complejos. Tales sistemas pueden ser de muy variada naturaleza; así, por ejemplo, está el sistema económico-administrativo, único objeto actual de la contabilidad, que pertenece al mundo social, y cuyo fin es la utilidad; el sistema moral, dentro del mundo social también, y cuyo fin es el bien; el sistema biológico, dentro del mundo físico, dirigido a conseguir una función biológica eficiente, etc.

Esta posición de proponer como objeto de conocimiento contable cualquier fenómeno natural o social se ha ido perfilando en los últimos años, a partir de la década de los sesenta del presente siglo, hacia la observación contable de los fenómenos circulatorios, económicos o no económicos, con base en el análisis circulatorio, metodología que remplazaría a la partida doble. El profesor Moisés García, investigador español de la Universidad Autónoma de Madrid, inicia su obra Contabilidad Social (1980), con los siguientes párrafos.

Por primera vez también se define el 'sistema de la circulación económica', con base al cual puede trasladarse al análisis económico todo el esquema del análisis circulatorio. El resultado es sorprendente por cuanto se abren insospechadas posibilidades al nuevo análisis contable en economía, superiores a las del convencional, basando en el armazón formal extremadamente simplista de la partida doble.

En lo macroeconómico, el razonamiento circulatorio arranca con las formulaciones de Quesnay (Tableau economique). Es ampliamente utilizado por Marx y reaparece posteriormente en los trabajos de Leontief y de Keynes. Las teorías de este último sobre el ingreso y gasto nacional agregados cristalizan finalmente en los modernos modelos de Cuentas Nacionales a través de los trabajos de Richard Stone. 
Sin embargo, no hay nada de economía en la 'teoría de las estructuras y sistemas circulatorios', fundamento lógico del análisis circulatorio y, por tanto, del análisis contable. Se trata de una teoría formal que hunde sus raíces en la tradición de las construcciones lógico-matemáticas, y cuyos antecedentes más próximos son las modernas 'Teoría de conjuntos' y la 'Teoría General de Sistemas'. Dentro de esta última, la 'Teoría de los modelos' resulta perfectamente aplicable a los modelos contables en tanto modelos de un tipo particular de sistemas: los sistemas circulatorios (García, 1980).

Con todos estos avances y aún más, considerando los nuevos retos aparecidos en el panorama contable, de informar sobre los fenómenos ambientales naturales y sociales diferentes de los tradicionales, la perspectiva contable de desarrollarse prácticamente en ámbitos que vayan más allá de lo meramente económico-financiero parece todavía lejana. Para constatar ello basta con observar los contenidos académicos dominantes en los programas de contaduría o ciencias empresariales, en los que nos encontramos con la escasa o nula presencia de planteamientos del tenor formal que acabamos de mencionar. Una causa explicativa de esta situación es el escaso desarrollo teórico de esta tendencia, la cual, salvo contadas y honrosas excepciones como las de los profesores Richard Mattessich, Moisés García, Yuri Ijiri y pocos más, no ha aportado una cantidad suficiente de obras que divulguen los modernos planteamientos con miras a solucionar los nuevos problemas contables.

En relación con el tiempo formalizador el profesor Montesinos cita a algunos autores del siglo XVII y del XIX como los pioneros de dicho enfoque, entre los que destacamos a Hustcraft Stephes, como iniciador en Inglaterra, en 1736, de la Teoría del propietario y quien formula sus explicaciones contables recurriendo a planteamientos algebraicos elementales pero muy significativos por su claridad y sencillez expositiva. Así mismo sus seguidores W. Fulton y W. Cronhelm, quienes publicaron sus obras en 1800 y 1818, profundizan en las explicaciones de la mecánica contable bajo la óptica matemática. También a Giovanni Rossi, apasionado seguidor de la obra de Cerboni, quien publicó en 1895 su Trattato della unitá teorética dei metodi discrittura doppia, y en 1901 la Teoría matemática della scrittura doppla italiana, en las que explica que "la cuenta puede 
considerarse como elemento matemático sobre el que pueden seguirse operaciones de cálculo, por cuanto está formado de dos series de cantidades homogéneas, aunque de significado opuesto y de signo contrario entre sí, esto es, una parte positiva y otra negativa, anotadas en las dos partes en que la cuenta está dividida" (Montesinos, 1978).

Afirma el profesor Montesinos que es a partir de los años cincuenta del siglo XX cuando irrumpe con fuerza la matemática en el terreno de las ciencias económicas y particularmente en la contabilidad. Las obras de Paton Accounting Theory de 1922; de Cutolo Sull atribuzione del valore del Neto nel Bilanci Contabile de 1952; de Palomba Introduzione all'Economica de 1950; de Devine Integration of Accounting and Economics in the Elementary Accounting Course de 1952; y de Littleton Accouting Rediscovered de 1958, constituyen una buena muestra de esta irrupción de la utilización de la herramienta matemática en la explicación de los planteamientos contables. Pero es la obra del profesor Richard Mattessich, iniciada con su escrito Towards a general and axiomatic foundation of accountancy aparecido en Accounting Resarch, de octubre de 1957 y continuada con Accounting and Analytical Methods en 1964 y otras publicaciones posteriores, las que propician definitivamente la calificación de la existencia de una escuela o corriente formalizadora de la contabilidad por autores como Leandro Cañibano en el sentido de programa de investigación apuntado por Lákatos.

Finaliza este acápite el profesor Montesinos (1978), con algunas precisiones de importancia para los estudiosos contables que se inician en su investigación, advirtiendo que

La formalización contable, como la de otras ciencias, debe contemplarse como la puesta en marcha de una simbolización lingüística precisa, mediante la cual nos sea posible ordenar y delimitar claramente las ideas de que disponemos, obteniendo el máximo fruto posible de las estructuras con las que trabajamos, y depurar los planteamientos; sería desacertado, sin embargo, pensar que la formalización es un instrumento o un método adecuado para la consecución de nuevos descubrimientos. La formalización en contabilidad es un fenómeno evidente para cualquiera que tome contacto con las publicaciones de la materia, en especial las anglosajonas. Su desarrollo se ha planteado, fundamentalmente, en las dos direcciones siguientes: a) Estudio del comportamiento de las 
magnitudes objeto de los estudios contables, a través de modelos formalizados que manifiesten de manera precisa las relaciones en las que aquellas intervienen. b) Investigación de las características generales de la estructura de los sistemas contables de información, en especial aquellos que se implantan con base en el sistema de la doble clasificación. En esta segunda dirección hay que resaltar los intentos de axiomatización realizados, en los cuales el de Mattessich es, sin duda alguna, el más perfeccionado y riguroso.

d) La inclusión de los aspectos conductistas y la perspectiva comunicacional. Según el profesor Montesinos (1978), la disciplina contable se ha visto influenciada por las modernas tendencias de los enfoques organizativos, tal como el enfoque decisional planteado en 1938 por Chester Barnard y continuado por H. Simon, J.G. March, R.M. Cyert, y C. Bonini. Desde esta perspectiva las organizaciones son coaliciones de individuos que persiguen sus propios objetivos y que se los traslada a la organización. La contabilidad también ha sido influenciada por esta tendencia y por ello, de un sistema dirigido a colaborar con la dirección en el proceso de maximización de beneficios a través de un sistema de evaluación que pretende ser neutral, pasa a percibirse como un sistema de información encaminado a facilitar datos en diversos niveles de la gestión, permitiendo así una toma de decisiones fundamentada, referida a las funciones de planificación y de control. Según este enfoque, la contabilidad debe servir como medio de comunicación, para ello el contador debe seleccionar, procesar y comunicar los datos relevantes para las diferentes decisiones, adoptando una visión integral de los fenómenos observados, contemplando sus diversos aspectos y las interacciones que puedan producirse entre las partes del sistema.

e) El enfoque económico. Finalmente, llegamos al enfoque económico, de fundamental interés para el presente recuento histórico, ya que a partir de él justificamos el entronque contabilidad-economía, base para la comprobación de la hipótesis de la investigación, relativa a la posibilidad de realizar un tránsito desde lo micro contable a lo macro contable. Obviamente que para la comprobación de la mencionada hipótesis tomaremos aportes realizados por autores clasificados en otras escuelas, como es el caso del trabajo de Mattessich. 
El profesor Montesinos, cuyo trabajo continuamos siguiendo en este apartado, propone una clasificación de diez tendencias de investigación, como componentes del enfoque económico. Haremos una breve referencia a algunas de ellas, las que en nuestro modesto criterio consideramos más relevantes.

- Controlismo

- La escuela de la Economía Hacendal

- La doctrina alemana de la Economía de la Empresa

- Patrimonialismo

- Contabilidad Social

- El Controlismo es una escuela veneciana encabezada por Fabio Besta, quien abandona el contismo y las posiciones cerbonianas y desarrolla un concepto de contabilidad entendida como ciencia del control económico lo que origina la denominación Controlismo.

Esta corriente entiende que el objeto de los estudios contables es el control económico de la riqueza hacendal. De forma más pura que los cerbonianos, se concentra más en la contabilidad y procura dar el máximo a la riqueza patrimonial como algo concreto, real, objeto de indagaciones propias, más que como finalidad de entenderse como la senda o el control.

La obra de Besta, de gran profundidad en el tratamiento de los temas, denota su principal preocupación: dar jerarquía y rango científico a la contabilidad. Basándose en el estudio histórico de la cuenta y teniendo presente la noción económica de valor, establece la definición de cuenta, así: "una serie de notaciones referidas a un objeto determinado conmensurable y mutable, efectuadas con la finalidad de registrar las condiciones y medidas de tal objeto en un instante dado y de registrar también las mutaciones que va experimentando, para poder dar razón del estado de este objeto en un determinado momento" (Montesinos, 1978).

Besta investiga las funciones de la administración económica, distinguiendo tres tipos de funciones diferentes: de gestión, de dirección y de control. Es en el tercer grupo donde la contabilidad centrará su atención. Dentro del control distingue dos partes: registro y constreñimiento del trabajo económico, clasificando luego las funciones de con- 
trol con respecto al tiempo en que acaecen los hechos administrativos a los que se refiere y con respecto a su naturaleza.

El Controlismo como tal no se desarrolla fuera de Italia y hoy en día se considera superado, aunque deja sentadas bases sobre las que se fundamentarán, más tarde, estudios de administración y organización de empresas.

- La escuela de la Economía Hacendal, fue creada y promovida por Gino Zappa, sucesor de Besta. Este autor define la Economía Hacendal como la ciencia que estudia las condiciones de existencia y las manifestaciones de vida de las haciendas, y dentro de ellas distingue tres disciplinas íntimamente relacionadas: organización, técnica administrativa y contabilidad. La obra de Zappa pone de relieve la conexión sistemática entre las tres disciplinas, hasta el punto que su desarrollo aislado no puede entenderse científicamente. En este punto su aportación es trascendental, por cuanto pone de manifiesto el carácter sistemático e interconectado de las investigaciones científicas en el campo de las actividades económicas de las haciendas.

Según el profesor Lopes de Sa, la corriente haciendalista dominó la Italia del siglo XX, pero terminó por no ofrecer un cuerpo de doctrina abarcador, de lógica profunda, competente para justificar el campo contable. Su visión de la contabilidad no convence y no consigue imponerse por falta de lógica científica capaz de justificar la dignidad y la autonomía necesarias. Para tal economía hacendal la contabilidad se limitaba a los 'levantamientos' o 'revelaciones' de riqueza, pero no presenta la esencia para justificar la clasificación.

- La doctrina alemana de la Economía de la Empresa desarrolla un enfoque contable de difícil distinción entre una y otra disciplina, luego de un proceso investigativo y de avances prácticos, en los albores del siglo presente. Se destacan entre los investigadores de esta escuela los eminentes autores Schmalenbach, Schmidt y Schneider.

Schmalenbach desarrolla la Teoría dinámica, cuyo significado apunta a otorgar igual o mayor importancia al balance de rédito o estado de resultados que al balance patrimonial. Según el mismo autor no se trata de una verdadera teoría de balances, y tampoco quiere pasar por ser el primero en dar importancia a un estado contable que fue suficiente- 
mente destacado por Stevin en el año 1600. El propósito de su trabajo queda claramente expuesto al inicio del prólogo, al decir: "este libro estudia el cierre del ejercicio de las empresas mercantiles".

Este autor precisa el significado de los dos estados contables, balance y resultados: el primero es un estado estático, orientado a mostrar una adecuada información sobre el estado patrimonial; el segundo es un estado dinámico, dirigido a informar sobre la eficiencia económica de la producción de la hacienda.

En la contabilidad por partida simple se averigua, de hecho, el beneficio exclusivamente con la ayuda del Balance... En la contabilidad por partida doble, el Balance dispone de un satélite que es la cuenta de Pérdidas y Ganancias, la cual se empareja siempre con el Balance, como una mano con la otra. La cuenta de Pérdidas y Ganancias muestra, en formas más o menos resumidas, los movimientos habidos durante un periodo contable, y el Balance presenta la situación resultante de esos movimientos (Montesinos, 1978).

Por su parte Schmidt, influenciado por criterios de la economía positiva, trata de reflejar estos puntos de vista en la contabilidad con el propósito de alcanzar una información 'verdadera' con respecto al patrimonio empresarial y al rédito del ejercicio, acción orientada a coadyuvar a la entidad en la búsqueda de conservar su valor-relativo en el ámbito económico donde participa, y en la obtención del mayor rédito posible del empleo del capital.

Erich Schneider es un seguidor de los dos autores antes mencionados y continuó su labor en la dirección planteada al interior de la Economía de la Empresa, fundamentando la información contable en sólidos criterios económicos, tanto del nivel de economía empresarial como de economía política.

La contabilidad no es para Schneider una técnica (la partida doble), ni una necesidad práctica para la vida administrativa de la empresa (contabilidad externa), ni siquiera un imprescindible método de control (contabilidad de costes). Es todo eso y algo más; es el instrumento sensible de los órdenes, desde el técnico de la producción hasta las previsiones de los precios y las expec- 
tativas del movimiento de los mercados, suministrando así la información necesaria en cada caso, para la perfecta marcha en la empresa (Montesinos, 1978).

- El Patrimonialismo fue fundado por el eminente investigador contable italiano Vicenzo Masi, seguidor de Besta, plantea que la contabilidad es la ciencia del patrimonio, orientación que ha sido adoptada por investigadores, no tanto de Italia como de otros países, como España, Brasil, Argentina, Uruguay y Colombia. En palabras del profesor brasileño Lopes de Sa significa:

"La corriente contable que tiene mayor repercusión en la actualidad es la patrimonialista... El patrimonialismo continúa como una corriente viva, avanzando cada vez más profundamente, presentando obras de raro valor científico y filosófico en muchos países del mundo. Los fenómenos patrimoniales, o sea, todo lo que acontece con la riqueza hacendal, continúa siendo objeto central de estudio de las mejores obras de nuestra tiempo y la propia parte instrumental, formal, escritural, prosigue siendo normalizada dentro de una óptica nítidamente patrominialista (Montesinos, 1978)".

Masi parcela el estudio de la disciplina contable en tres componentes: la estática patrimonial, la dinámica patrimonial, y la revelación del patrimonio.

- La estática es el estudio del patrimonio en un momento dado del tiempo, como si su acción estuviera detenida, para examinar cuantitativa y cualitativamente su composición, sus elementos, cantidades, valores, estructura y condiciones de equilibrio.

- La dinámica es el estudio del patrimonio como un todo en movimiento continuo, causado por factores internos y externos a la organización. Examina el patrimonio, las inversiones y las financiaciones y los movimientos que los afectan reflejados en los ingresos, costos y gastos, entradas y salidas financieras, económicas y patrimoniales, relativas a las organizaciones institucionales.

- En cuanto a la revelación dice el profesor Masi: "La estática y la dinámica patrimonial no podrían ser estudiadas totalmente ni 
explicadas completamente, sin el uso de procedimientos diversos, oportunamente coordinados, de revelaciones que se evidenciaran en el patrimonio y los hechos patrimoniales, tal y como se manifiestan en las empresas y en las instituciones de todas las naturalezas, y así mismo justifica la existencia de una revelación patrimonial o levantamiento patrimonial".

- Por Contabilidad Social se reconoce la tendencia que se ocupa de reflejar los fenómenos macroeconómicos. También se la ha denominado contabilidad macroeconómica, macrocontabilidad o cuentas nacionales.

Según el profesor Adolf Enthoven se pueden diferenciar tres etapas en el desarrollo de la contabilidad social, así:

a) Primera fase, que data desde la antigüedad hasta 1930, periodo en el que se van perfilando las ideas en torno al proceso de circulación de valores en las economías y aparecen estimaciones de la renta en casi todos los países, de una forma o de otra. En esta etapa se destacan los trabajos de Sir William Petty (1623-1687), Richard Cantillon (1680-1734), Francoise Quesnay (1694-1774). La idea de la circulación de valores dentro del sistema económico considerado como un conjunto de partes interrelacionadas se desarrolla a través de los trabajos de Karl Marx, León Walras y Wilfredo Pareto.

b) Segunda fase, que se desarrolla entre 1930 y 1945, época de crisis económica en que los estudios teóricos y las realizaciones prácticas sobre Contabilidad Nacional se ven influenciados por la obra de Keynes. En esta etapa se inscriben las obras de Ragnar Frish, Richard Stone, Ed Van Cleeff, Jan Tinbergen y Wassily Leontief.

c) La tercera fase se desarrolla a partir de 1945 hasta nuestros días y es la de mayor ímpetu en el auge de la Contabilidad Social. A partir de la finalización de la segunda Guerra Mundial, a instancias de la Organización de las Naciones Unidas, se inicia los primeros estudios sobre las estadísticas de la renta nacional. En 1947 se publican los resultados de estas investigaciones, incluyendo diversas fórmulas para la totalidad del sistema económico en un marco de contabilidad nacional. 


\section{La perspectiva de Cañibano y Gonzalo}

Por su parte el profesor Leandro Cañibano (1974) identifica tres programas a partir de la publicación de la obra pionera de la reflexión teórica contable por Paciolo en 1494.

El primer programa con una vigencia aproximada entre 1494 y 1914 lo denomina Legalista, pues dice él:

La finalidad de la información contable entra dentro de unos causes estrictamente jurídicos y, por tanto, hay que construir todo el armazón atendiendo a las limitaciones derivadas de tal contexto. La objetividad de la información contable se entiende en sentido eminentemente legalista, así toda representación y valoración contable ha de estar sustentada sobre hechos o sucesos que, a efectos legales, pueden ser considerados como prueba, ya que lo que priva la expresión del patrimonio en términos cuantitativos no puede apartarse del concepto jurídico del mismo, en tanto la finalidad atribuida a la información contable consiste en la presentación de unos datos que sirvan de garantía a terceras personas (Cañibano, 1974).

Aunque éste no era el objetivo único de la contabilidad aplicada en este tiempo, pues desde las primeras formulaciones contables ha permanecido subyacente el objetivo de información económica, frente al legalista, aquél queda anulado.

Transformaciones socioeconómicas originadas en la primera posguerra mundial, como la inflación galopante en Alemania, posibilitaron el cambio de óptica en el orden de la importancia de estos dos objetivos de la información contable. La orientación legalista y su derivación, la rigurosa objetividad legal, no permitían valorar la realidad económica en su dinámica cambiante.

Nos encontramos, pues, con unas circunstancias que alteran totalmente el papel de la información contable; se le pide que sea capaz de ofrecer una base de cifras realistas, que se adapte al medio, que los resultados calculados respondan a principios económicos, que su conocimiento verdadero evite la creciente descapitalización de las empresas, en suma, que el fin que antes perma- 
necía en un modesto segundo plano surge con fuerza arrolladora sobrepasando al que hasta ese momento ocupaba total prioridad (Cañibano, 1974).

Así aparece y se desarrolla, primero en Alemania y luego se irradia en el ámbito internacional el Programa Económico, el segundo que identifica Cañibano cuya vigencia dominante la ubica entre 1914 y 1960. Dentro de este enfoque se desarrollan conceptos valorativos como los de costo de reposición, resultados exógenos y good-will, y se lanza un nuevo concepto que apunta a la homogeneización de criterios en el proceso de medición e información contable como es el plan de cuentas, hoy por hoy de amplísima aceptación en el continente europeo y en el ámbito latinoamericano, soporte del encuentro entre las micro y macro magnitudes contables.

En la segunda posguerra se vive un auge de las aplicaciones matemáticas para solucionar problemas científicos y técnicos. La contabilidad no es ajena a dicha influencia. Así se desarrollan los costos estándar y técnicas propuestas basadas en formulaciones matemáticas y estadísticas resueltas con ayuda del computador. La aplicación de la investigación operativa a las ciencias económicas pronto influye a la contabilidad y contribuye a que las exigencias informativas de las empresas comiencen a ser respondidas con mayores perspectivas de acierto en relación con las predicciones que se realizaban cuando no se hacía uso de esta herramienta.

Cañibano dice que si bien es cierto que la puesta en práctica de dichos modelos hubiera sido imposible en otras épocas, a partir del momento al que nos referimos comienza una verdadera revolución en el campo de la informática y de la cibernética; el diseño de nuevos ordenadores, cada vez más poderosos, hace factible el tratamiento de modelos matemáticos aplicados a las ciencias empresariales, dentro de ellas a la contabilidad.

Sin embargo, en contabilidad se presentaba un vacío en el campo de los estudios metodológicos, pues los intereses de los estudiosos, hasta bien entrado el presente siglo, se habían orientado hacia derroteros pragmáticos. Esta falencia hacía que cualquier mejora en la parte mecánica perdiera su efectividad, en tanto no hubiera un cuerpo teórico formalizado que permitiera establecer unas bases generales sobre las 
que se fundamentan las aplicaciones particulares en cuya práctica se desarrollarían.

Como respuesta a esta necesidad, comienzan a aparecer los primeros intentos de axiomatización contable con los trabajos de Paton en los años 20 y de Paton y Littleton en los 40.

No puede hablarse seriamente de dicha preocupación hasta que ve la luz el articulo de Richard Mattessich Hacia una fundamentación general y axiomática de la contabilidad publicado en 1957, en el cual se establece una serie de axiomas, definiciones, teoremas y requisitos, apoyándose en el álgebra matricial como lenguaje formal. Sin embargo, la formalización rigurosa de la teoría quedaría aplazada hasta 1964, año en que es publicada otra obra del profesor Mattessich Contabilidad y métodos analíticos. Medición y proyección del ingreso y la riqueza en la microeconomía y la macroeconomía, en cuyo apéndice es presentado a escala formal mediante la teoría de conjuntos el cuerpo de fundamentos sobre los que descansa la teoría de la contabilidad, los cuales son desarrollados magistralmente a nivel semántico a lo largo de toda la obra. La rigurosidad formal permite un análisis de gran profundidad de aspectos de la mayor relevancia, tales como medición, valoración y predicción (Cañibano, 1974).

Esta orientación motivó el interés de una gran cantidad de estudiosos de la teoría contable a partir de los años 60, etapa que al profesor Cañibano le ha permitido identificar como la del surgimiento de un nuevo programa de investigación que él ha denominado Programa Formalizado, cuyo objetivo se dirige a "la reducción de las proposiciones contables a cálculos lógicos o matemáticos, para, en virtud de las reglas de inferencia, llegar a unos resultados capaces de ser interpretados semánticamente, y cuyo contraste con la realidad irá elevando, de día en día, su potencialidad explicativa y predictiva" (Cañibano, 1974).

Esta metodología de reconstrucción histórica del quehacer contable ha sido también aplicada por el profesor Jorge Tua (1984), para describir el proceso de formulación doctrinal y de regulación contable en Estados Unidos de Norteamérica. Tua identifica dentro del Programa Formalizado tres subprogramas de investigación así: un periodo previo de formación doctrinal y acción regulativa denominado subprograma 
de búsqueda y dos periodos sucesivos, uno sustentado en la lógica clásica, predominantemente cognoscitiva, denominado subprograma lógico y el otro, el subprograma teleológico, impulsado por el criterio de utilidad de la información que puede, según Tua, desembocar en construcciones decididamente normativas y deónticas.

Estas características o rasgos básicos de ningún modo se abandonan al variar los enfoques, lo que sucede es que cambian de lugar en cuanto a las prioridades y siguen influyendo de una u otra forma en las diferentes etapas.

Para el subprograma de búsqueda, según Tua (1984), las reglas contables son fruto de la experiencia más que de la lógica.

Un buen número de autores que se ha ocupado de los primeros pasos de la regulación indica que toda disciplina científica nace normalmente como un conjunto de procedimientos para resolver una serie de necesidades específicas y encuentra inicialmente una vía de apoyo en la propia práctica más que en la reflexión semántica. A medida que se produce su desarrollo, se van realizando construcciones encaminadas a formalizar, explicar y predecir el comportamiento de los hechos, que normalmente contribuyen a mejorar sus aplicaciones prácticas (Tua, 1984).

Con este criterio de base, este periodo previo a la formalización del discurso contable se caracterizó por aplicar una metodología inductiva dirigida a identificar las prácticas en uso, agruparlas alrededor de unos criterios y objetivos básicos para con ellos establecer un conjunto regulativo con pretendida validez general. De ahí que esta etapa también es conocida como de aceptación generalizada.

Comúnmente se ha definido al método inductivo como el procedimiento que permite extraer conclusiones generales de observaciones y mediciones particulares; esta orientación guió a los estudiosos de la contabilidad en EE.UU. durante la época de la Gran Depresión hasta la década de los 50. Para el medio contable colombiano es de gran interés conocer este proceso debido a la marcada influencia que sus contenidos doctrinales y regulativos han tenido en la práctica contable de nuestro país. Este ensayo investigativo siguió la construcción inductivo-positiva, caracterizada por cuatro etapas así: a) Observación y registro de todos los hechos; b) Análisis y clasificación de éstos; c) Derivación in- 
ductiva de generalizaciones; y d) Contrastación ulterior de las generalizaciones. Postulando, por otro lado, que las hipótesis de trabajo no aparecen en esta secuencia sino hasta su tercer estadio, es decir, el de la derivación inductiva de generalizaciones, con el fin de garantizar la asepsia necesaria, desde la corriente positivista, en la cual se postula que si hay preconceptos, esto es, juicios de valor, se invalida el proceso investigativo, pues sus resultados estarían viciados.

Sin embargo, como dice Tua, la determinación de qué hechos de los observados son relevantes al momento del estudio propuesto y la elección a favor de una taxonomía con respecto a otra parece confirmar que el tipo de datos que se hayan de reunir o clasificar no está determinado por el problema que se está estudiando, sino por el interior de respuesta que el investigador trate de darle en forma de conjetura o hipótesis. Es la posición subyacente de la mente de quien opera, en esencia sus propios juicios de valor la que establece la relevancia o irrelevancia de los datos en función de las hipótesis previas que maneje.

Otro problema que incide en esta forma metodológica de proceder es el identificado por Hume y objeto de muchos análisis, entre ellos, de Popper en varias de sus obras. Popper responde a este problema con su propuesta metodológica de la falsación como remplazo a la verificación inductiva.

En el caso del subprograma de búsqueda las falencias anotadas explicaron el origen de múltiples problemas en el proceso práctico que impedían dar respuesta adecuada a las crecientes necesidades informativas. Los teóricos de la contabilidad destacaron estos puntos débiles que fueron ocasionados por el pragmatismo a ultranza. Con él, la general aceptación pierde todo soporte que no fuese la práctica, con lo cual aparece la clásica trampa teleológica: se confunde la causa con el efecto y la norma racionalmente fundada se convierte en uso, y en lo que debe hacerse por lo que es y lo que se hace.

Tua ubica este momento de decadencia del subprograma de búsqueda hacia finales de los años 50 y comienzos de los años 60, cuando se crea un organismo en EE.UU. orientado a la investigación de las bases teóricas de la contabilidad, el Consejo de Principios Contables (A.P.B.). Aunque desde años atrás la doctrina contable había abordado dicho problema ahora se trata de darle un tinte de autoridad oficial a la producción teórica. 
"En este esquema se insiste en el desarrollo lógico contable en varios niveles, así como en la necesidad de una continua investigación. Su contenido marca una clara impronta en la época, fijando una tendencia direccional posterior, un subprograma de investigación en suma, de manera que las declaraciones doctrinales y profesionales que le sucedieron siguieron sus expresiones y conceptos, consagrando el esquema postulados-principios-reglas, utilizado en los trabajos de investigación ARS 1 y 3" (Tua, 1984), que se constituyeron, en un principio, en la orientación oficial por excelencia del Consejo.

El párrafo siguiente tomado del informe del comité especial de investigación, constituye el núcleo central en torno al que se mueve la heurística positiva del nuevo programa denominado subprograma lógico:

Los postulados son pocos en número y son presupuestos básicos sobre los que descansan los principios. Necesariamente derivan del entorno económico y político, y de las formas de pensamiento y hábitos de los diferentes segmentos de la comunidad de negocios. La profesión, no obstante, debe conocer claramente su significado e interpretación, al objeto de que constituyan una base significativa para la formulación de principios y para el desarrollo de reglas y otras guías para la aplicación de principios a situaciones específicas... Un amplio conjunto de principios contables coordinados debe servir como un entramado de referencia para la solución de problemas concretos. En consecuencia, las reglas y otras guías para la aplicación de principios contables a situaciones específicas deben desarrollarse en relación con los postulados y principios previamente expresados. Deben ser razonablemente flexibles (Tua, 1984).

Tua resalta de esta etapa dos elementos interesantes desde el punto de vista formal del contenido. En primer término el apego al esquema postulados-principios-normas como clara intención de adoptar la vía hipotético-deductiva para fundamentar la teoría contable. En segundo lugar se advierte, no explícitamente, una tendencia que servirá a partir de este momento como vía de solución en el permanente conflicto que existe entre las posiciones positivas y normativas. Nos referimos a la distinción entre teoría general contable y sus aplicaciones. La primera, como su nombre lo indica, general o común para cualquier desarrollo 
específico, establece las condiciones iniciales, además, no dependen del entorno, es decir, que son válidas para cualquier medio económico. Las segundas son aquellas que se adaptan al entorno específico en que van a servir de base para el desarrollo de políticas particulares y a la obtención de ciertos objetivos en una clara orientación teleológica.

El profesor Tua afirma que se desarrolla en esta época una etapa de producción teórica en la vía de la teoría básica tan fecunda, que ha sido denominada por Nelson la edad de oro de la investigación a priori. Agrega el profesor que un verdadero aluvión de trabajos saturó de hipótesis a priori la década de los 60, en la vía postulados-principios-normas con objetivos como los de construir estructuras plenamente integradas de teoría de contabilidad basadas en un conjunto dado de premisas o postulados; desarrollo de teorías sobre la medición contable, teoría de los costos y los valores; desarrollos matemático-contables, etc.

Paralelamente al subprograma lógico se desarrolla una tendencia investigativa crítica del mismo como réplica de la etapa primera, aparentemente ya superada, la de búsqueda, caracterizada por su orientación inductivo-positiva.

En el subprograma lógico la contabilidad se orienta hacia la búsqueda de una verdad única, el cálculo del beneficio y del patrimonio, sin importar quien lo recibe y por qué. La contabilidad buscó y tomó conceptos fundamentales basados en la teoría económica, especialmente en la microeconomía, suponiendo que con ello se facilitaría aquel cálculo y, por ende, la distribución de los recursos, con lo que los autores que han militado bajo este subprograma se orientaban más hacia la búsqueda de la verdad que a la noción de utilidad.

La gran cantidad de escritos en la vía apriorística saturó el medio académico contable. Este hecho, junto a la actividad crítica de los investigadores inductivo-positivistas, propició el surgimiento de un nuevo subprograma de investigación que se formalizó a comienzo de los años 70 y que Tua ha denominado subprograma teleológico. Dice Tua (1984) al respecto:

La preocupación por dotar a la norma contable de un sentido teórico, vuelve a hacer su aparición al comienzo de esta etapa, pero con un cariz diferente: en esta ocasión se trata de derivar tales estándares a partir de los objetivos de la información contable. Este 
nuevo enfoque que hemos denominado teleológico puede contribuir a la creación de una teoría normativa del estándar contable, en cuanto la selección de un conjunto de objetivos es inherente a la utilización de juicios de valor (Tua, 1984).

La heurística de este subprograma está soportada en la búsqueda de unos estándares o convenciones que apunten al alcance de unos objetivos considerados deseables para los estados financieros y la información contable en la perspectiva fundamentalmente del inversor bursátil.

Ya no se habla de principios sino de estándares o convenciones y la preocupación por encontrar una información única y verdadera, clásica de la epata lógica, es remplazada por la búsqueda de una información válida y útil al usuario.

Se nota, además, que se da una separación entre el concepto de teoría general y sus aplicaciones, con clara influencia del sector profesional que pretende obtener una regulación contable que responda a los intereses del medio usuario empresarial y del mercado bursátil, antes que profundizar en cuestiones teóricas más apropiadas de ambientes académicos en quienes parecen delegar esta tarea.

Así, el subprograma teleológico se desarrolla fundamentalmente en la línea pragmática en responder a las necesidades de los usuarios de la información, orientándose por un único esquema de reglas. Los usuarios de la información pueden clasificarse para el programa en dos grandes grupos a quienes se pretende satisfacer, así:

1. Los inversionistas bursátiles, como consumidores de información destinada a la toma racional de decisiones económicas, estudiando sus requerimientos y reacciones a nivel individual o colectivo, así como sus procesos decisorios, para derivar de los mismos las reglas que doten a la información contable la configuración que sea más adecuada a tales propósitos.

2. La entidad emisora y sus directivos, cuyas intenciones y objetivos se ven afectados por la utilización de la norma contable.

El profesor Richard Mattessich escribió, en 1986, el artículo Un examen científico aplicado para una estructura metodológica en el que incluye su visión del progreso investigativo de la disciplina contable, a 
partir de los planteamientos epistemológicos de Lákatos, Balzer, Stegmüller y Bunge. En el texto examina cómo las ideas de estos epistemólogos pueden ser aplicadas, de algún modo significativas, a la moderna teoría contable y al análisis de la información.

En este artículo el profesor Mattessich propone una agrupación de la reciente actividad investigativa contable, en tres tradiciones o programas, así: la tradición investigativa de gerencia, la tradición investigativa de valuación-inversión, y la tradición investigativa de información-estrategia.

La tradición investigativa de Gerencia, referida a la relación principal-agente y a la supervisión que el primero realiza sobre el segundo en su relación contractual, está conformada por tres propuestas, a saber, de periodización, original de la agencia y agencia-información.

La propuesta de periodización nace teniendo en cuenta que el monitoreo de la gerencia directiva puede ser satisfecho mejor con la apropiada asignación de costos y beneficios de inversión a un periodo específico. Está basada en los siguientes principios:

1. El costo histórico o costo de adquisición.

2. Asignación periódica de costos y beneficios a través de su comparación.

3. La asignación de los costos por depreciación con base en una vida útil estimada.

4. La suposición de la empresa marcha.

5. La función de gerencia que domina a esta fase.

Una característica fundamental de esta fase es que la función de valuación no es considerada como función contable, sino del mercado.

La propuesta original de la agencia comienza en el pionero trabajo de Coase de 1937 y en el de Simon de 1951, en los que se analizan sistemáticamente los problemas de los contratos de trabajo y dirección, planteamientos que tuvieron que esperar durante más de dos décadas para que fueran aceptadas como teorías válidas para estudiar las relaciones del principal y el agente. El problema nodal que aborda esta propuesta está referido a los costos generados en la conflictiva relación principal-agente, ocasionados en el control de la actividad del agente realizada por el principal, reducción de los resultados debido a dife- 
rencias de criterio en la valuación de diversas partidas, anticipación de acciones del agente en su favor frente a los intereses del principal. En relación con este punto se halla la búsqueda de un contrato que posibilite un reparto de riesgos entre el principal y el agente que pueda cumplir con el criterio de la optimalidad paretiana. Así el sistema de información contable adquiere una importancia vital, pues es la base para determinar el comportamiento del agente que no puede ser vigilado permanentemente por el principal.

La tercera fase de la tradición de gerencia es la propuesta agencia-información, y surge de la combinación de la teoría descriptiva de la agencia, por una parte, y la economía informática, por la otra. Aunque estos dos aspectos no estuvieron por muchos años relacionados, la creciente formalización de los aspectos precedentes facilitó su encuentro, lo que propició la estrecha cooperación en el proceso investigativo. Su núcleo debe encontrarse en las relaciones contractuales y en la participación en el riesgo entre el principal y el agente.

En la relación contractual entre el principal y el agente se pueden dar una amplia gama de modalidades que estarían contenidas entre dos extremos, uno de los cuales presenta al principal asumiendo el riesgo total y recibiendo el resultado remanente, y al agente, con riesgo cero y remuneración fija; el otro extremo presenta al principal recibiendo una remuneración fija y asumiendo cero riesgo, en tanto que el agente asume todo el riesgo y se embolsa el resultado restante. Entre las múltiples posibilidades contractuales las dos partes se orientan a buscar una que cumpla con la condición óptima paretiana en cuanto al resultado, y de acuerdo con la teoría económica marginal clásica, en relación con la participación en el riesgo.

El profesor Mattessich divide la segunda tradición investigativa ( $v a-$ luación-inversión) en tres propuestas, así: del valor presente-valor corriente, del riesgo compartido, y del mercado de capitales. El propósito fundamental de esta tendencia es la de aproximar a la contabilidad hacia la correcta valuación económica de los recursos y derechos.

La primera fase de esta tendencia, del valor presente y valor corriente está suscitada en la oposición al costo histórico y al costo acumulativo. Su comienzo se remonta al siglo XIX y comienzos del XX. Autores como Schmidt y Canning, en la década del veinte del pasado siglo, proponen alternativas como el valor de remplazo (valor 
corriente de entrada) para alcanzar de manera objetiva el valor económico actual. Además, se incluyen en esta fase propuestas de Staubus, Edwards y Bell, Sprouse y Moonitz, Chambers, entre otras, que plantean la adopción de sistemas de valoración concomitantes con los del valor corriente. Por otro lado y en la línea del valor presente, basado en la teoría del capital, se consideran los escritos de Hansen, Honko, Albach y Sich.

La segunda fase del programa de valuación-inversión es la propuesta del riesgo compartido. Esta fase también se basa en la teoría económica neoclásica y su extensión realizada por Arrow y Debreu, en 1954, pero principalmente en la moderna teoría del portafolio de Markowitz, expuesta por éste en 1952 y por Fama, en 1965, así como en la teoría de la cotización de activos de capital de Sharpe y Linther. Enfatiza la valuación de las predicciones del mercado e informaciones de carácter similar, así como sus efectos sobre la disciplina contable. Incluye procesos estocásticos en la contabilidad y toma en consideración la valuación de títulos en concordancia con la moderna teoría financiera con su distinción entre riesgos de mercado, el que puede ser eliminado por la diversificación del portafolio, por una parte, y riesgo sistemático para títulos que cambien más rápido y para títulos cuyos precios cambien más lentamente que el índice del mercado de valores, por la otra. Debe ser tenido en cuenta en esta fase, el trabajo de Ball y Brown publicado en 1968, pues fue la primera publicación que consideró la correlación entre los beneficios publicados y los precios del stock de títulos.

La tercera fase de la tradición valuación-inversión se denomina mercado de capitales. Está vinculada a la fase precedente y tiene similares premisas básicas, pero enriquecidas con la aceptación de que el beneficio pertinente de los títulos posee una genuina probabilidad de distribución. Se considera que la información del mercado tiene valor si ayuda a lograr la estimación del beneficio real. Así mismo se acepta la hipótesis de la eficiencia del mercado, con especial atención a la información del ámbito financiero.

La tercera tradición investigativa en la propuesta del profesor Richard Mattessich se denomina Información-estrategia y corresponde a una particular visión planteada en la década del veinte por J.M. Clark y que ha sido respaldada por él desde los años sesenta. 
Se plantean dos etapas en esta tendencia o tradición, así:

a) Formulación de las consideraciones básicas de la contabilidad como las puestas en práctica en el micro y en el macro nivel.

b) Distinción estricta entre teoría básica no interpretada o semi-interpretada y las interpretaciones orientadas a fines específicos, para diferentes aplicaciones.

En la ciencia moderna, dice Mattessich, se acepta en forma general la inclusión de hipótesis adicionales auxiliares para el caso de nuevas aplicaciones de una teoría básica.

\section{Bibliografía}

D’Auria, Francisco. (1949). Primeiros principios de contabilidade pura. Universidade de Sao Paulo, Sao Paulo.

García, Moisés. (1980). Contabilidad social. Del sistema de la circulación económica a los modelos de cuentas nacionales. Madrid: Ministerio de Hacienda.

Matтessich, Richard. (1986). Un examen científico aplicado para una estructura metodológica. Revista Teuken $N^{\circ} 3$, tercer trimestre de 1988. Universidad Nacional San de la Patagonia Juan Bosco de la Patagonia, Comodoro Rivadavia, Argentina.

Montesinos, Julve. (1978). Formación histórica, corrientes doctrinales y programas de investigación en contabilidad. Técnica Contable t. XXX. Madrid.

CAÑibano, Leandro. (1974). Teoría actual da la contabilidad. Madrid: Editorial ICE.

Cañibano, Leandro y Gonzalo, José Antonio. (1995). Los programas de investigación en contabilidad. Primeras Jornadas de Teoría de la Contabilidad, Jeréz, España.

TuA, Jorge. (1984). Principios y normas de contabilidad. Madrid: Instituto de Planificación Contable. 


\title{
HÁROLD ÁLVAREZ ÁLVAREZ
}

\author{
alye@une.net.co
}

Contador Público (Universidad Nacional de Colombia, Bogotá). Magíster en Ciencias Financieras y Sistemas (Universidad Central, Bogotá). Estudios Doctorales en Economía Empresarial (Universidad Autónoma de Madrid, España). Expresidente del Consejo Técnico de la Contaduría Pública. Académico del Centro Colombiano de Investigación Contable (CCINCO). Articulista en revistas académicas especializadas del orden nacional e internacional. Profesor universitario, de pregrado y postgrado en la Universidad del Quindío, Universidad de Manizales, Universidad la Gran Colombia, entre otras. Actualmente es docente e investigador de la Universidad Autónoma Latinoamericana y de la Universidad de Manizales. Su interés académico ha girado en torno a temas como teoría contable, contabilidad internacional, y la relación contabilidad y economía. 